\title{
Editorial PIAM April 2019
}

\section{Christian Seidel $^{1}$}

Published online: 10 May 2019

(c) Springer Nature Switzerland AG 2019

Since its release in 2016, I have been a member of the Editorial Board "Progress in Additive Manufacturing". It is impressive how both the journal itself and our additive manufacturing industry have developed. According to latest State of the Industry reports we are approaching a total market volume of 10 billion US \$ annually. Especially in selected industries, such as aerospace, medical and even automotive, we observe an increasing amount of serial application use cases. For this reason, the demand for scientific articles in the field of Additive Manufacturing is constantly increasing. Focusing on high quality manuscripts, we try to meet this increasing demand with our scientific journal "Progress in Additive Manufacturing".

As member of the Editorial Board, one of my key roles is to ensure that manuscripts in our system are properly vetted by a minimum of two reviewers in a blinded review process. A blind review means that the identities of authors and reviewers are concealed from each other throughout the review process. It is thus important that authors should not indicate their affiliation within the manuscript and papers published by the authors should be cited anonymously. In addition, any funding sources and acknowledgements have to be removed.

A major improvement to reduce the peer-review time was the implementation of classification of manuscripts in which during the submission process, the Editorial Manager system requires authors to indicate the scope of their paper based on at least ten different classifications from the field of Additive Manufacturing, ranging from terminology, methods and processes, feedstock materials, finished parts and test methods, data, design, equipment, applications, environment, health and safety, training, education and standards. In addition to manuscript classifications, reviewers have been also asked to classify themselves according to their area of expertise. This ensures that the review process will be able to more accurately match the professional background of the reviewers according to the scope of the paper. To further reduce the peer-review time, auto reminders have now been set up so that our handling editors and reviewers have a time limit to complete their review. The new Editorial Manager version 15.0 has an improved author submission interface to make the manuscript submission process quicker and easier for authors.

Most of our latest changes implemented for Progress in Additive Manufacturing have been developed by working closely with authors and readers. There are further enhancements planned to streamline our publication process and to provide our authors with the best possible processes and software tools for paper submission. If you have any ideas for improvements please feel free to contact myself, my colleagues at the Editorial Board or Springer staff at any time.

Thank you for making PIAM a valuable source of information for our Additive Manufacturing industry. I hope you enjoy reading our current issue and that you will find a lot of valuable information for your work.

Christian Seidel

Member of the Editorial Board

Progress in Additive Manufacturing Journal April 2019

Publisher's Note Springer Nature remains neutral with regard to jurisdictional claims in published maps and institutional affiliations.
Christian Seidel

christian.seidel@igcv.fraunhofer.de

1 Fraunhofer IGCV, Augsburg, Germany 